\title{
MULTIVARIABLE CONTROLLER TUNING
}

\author{
Karl H. Johansson’, Ben James, Greyham F. Bryant,
}

\begin{abstract}
The problem of tuning individual loops in a multivariable controller is investigated. It is shown how the performance of a specific loop relates to a row in the controller matrix. Several interpretations of this relation are given. An algorithm is also presented that estimates the model required for the tuning via a relay feedback experiment. The algorithm does not need any prior information about the system or the controller. The results are illustrated by an example.
\end{abstract}

\section{Introduction}

Poorly tuned control loops represent a large economic cost for industry [5, 4]. Control parameters are often set to default values or are manually tuned in an ad hoc way. The reason for this is that there is a great lack of tools for tuning industrial controllers systematically. Nowadays there exist methods for automatic tuning of SISO control loops, which have been widely accepted and implemented in several commercial controllers [2]. Many control loops are, however, coupled and the interaction has to be considered in the control design to gain improved performance [18]. Most modern multivariable control design methods require a full model of the process [11]. In many cases such a model is not available and physical modeling or system identification may require a prohibitive engineering effort. Furthermore, it is hard, or impossible, to impose a certain control structure on standard multivariable design methods. Therefore, there is a need for simple methods of tuning multivariable controllers; particularly methods that compromise optimality for engineering efficiency.

This paper focus on the problem of retuning an existing multivariable control system. A framework is developed where it is possible to derive the influence of retuning one loop on the overall closed-loop performance. A badly tuned loop can in this way be improved by changing certain elements of the controller matrix. Tuning a loop corresponds to changing a row in the

\footnotetext{
$\dagger$ Department of Automatic Control, Lund Institute of Technology, Box 118, S-221 00 Lund, Sweden, \{kalle,kja\}@control.1th.se.

‡Exposure Management, Bank of America, Elmfield Road, Bromley, London, United Kingdom, 113277.2673@compuserve.com.

$\S$ Industrial Systems Group, Centre for Process Systems Engineering, Imperial College of Science, Technology and Medicine, Exhibition Road, London, SW7 BT, United Kingdom, g.bryant@ic.ac.uk.
}

controller matrix; hence, to solve a SIMO control design problem. Several quantities useful for estimating the influence of a controller row on the closed-loop system are derived. The information required for this type of design is also discussed together with how this information can be obtained. It is shown that no prior knowledge of the process dynamics or of the controller dynamics is needed, if a modeling experiment based on relay feedback is used.

In existing work on extending the auto-tuning method for SISO control systems developed in [1] to MIMO systems, either one relay is used for each experiment by closing one loop at a time $[7,6,19,17]$ or all loops are set under relay feedback simultaneously [23, 19, $12,20]$. A major drawback with the latter approach is that instead of giving stationary limit cycles the relays can induce very complicated oscillations $[19,9]$. There exist no results in terms of plant data for when this may or may not happen. Based on a successful relay experiment a controller is designed. Most authors limit the control structure to a decentralized configuration of SISO PID controllers [21, 19, 23, 17, 12]. Decoupling design is derived in $[6,20]$. Tuning cascade controllers (MISO controllers) is considered in [7, 22]. For a survey on relay feedback methods see [3, 9].

The outline of the paper is as follows. Section 2 presents some results that are useful for loop tuning. Retuning a row in the controller matrix is formalized. In Section 3 it is shown that the required information about the system can be obtained from an experiment with SISO relay feedback. Section 4 describes an application to a model of a new laboratory process. Some concluding remarks are given in Section 5. An extended version of this paper is given in [9].

\section{Loop Tuning}

Suppose that a multivariable control system with unsatisfactory closed-loop performance is given. The basic idea is to adjust certain elements of the controller matrix in order to improve the closed-loop behavior. In general, such an adjustment will affect all loops in the system. The challenge is to obtain this effect on the desired loop without degrading the performance of the other loops. This section gives results which enables the designer to compute the effect of an adjustment of a single loop on the overall closed-loop behavior. 


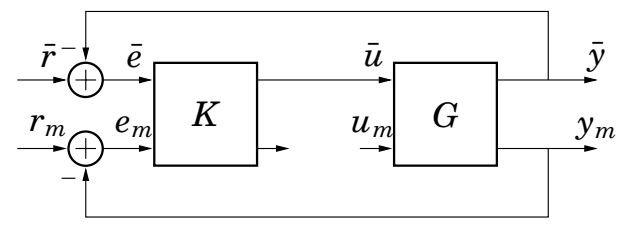

Figure 1 Opening of control loop $m$.

\section{Notation}

Assume that there exists a stable closed-loop system comprising a process $G$ and a nominal controller $K$, both with $m$ inputs and $m$ outputs. Denote the manipulated variable or process input $u=\left(u_{1}, \ldots, u_{m}\right)^{T}$, the controlled variable or process output $y=\left(y_{1}, \ldots, y_{m}\right)^{T}$, and the reference or set-point $r=\left(r_{1}, \ldots, r_{m}\right)^{T}$. The controller matrix $K$ acts on the error signal $e=$ $\left(e_{1}, \ldots, e_{m}\right)^{T}=r-y$. Hence, $y=G u$ and $u=K e$. The aim of the tuning procedure is to improve the performance of one loop by adjusting appropriate elements of the controller matrix. Without loss of generality, consider loop $m$ and define the following partitions:

$$
G=m\left(\begin{array}{cc}
G_{1} & G_{2}
\end{array}\right), \quad K={ }^{m-1}\left(\begin{array}{c}
K_{1} \\
k
\end{array}\right) .
$$

Partition the vectors $u=\left(\bar{u}^{T}, u_{m}\right)^{T}, y=\left(\bar{y}^{T}, y_{m}\right)^{T}$, $r=\left(\bar{r}^{T}, r_{m}\right)^{T}$, and $e=\left(\bar{e}^{T}, e_{m}\right)^{T}$ correspondingly, so that $\bar{u}=\left(u_{1}, \ldots, u_{m-1}\right)^{T}$ etc. Then

$$
u_{m}=\varepsilon_{m}^{T} K e=k e=k_{1} e_{1}+\cdots+k_{m} e_{m},
$$

where $\varepsilon_{m}^{T}=(0, \ldots, 0,1)$ and $k_{i}, i=1, \ldots, m$, are the elements of $k$. Row $m$ of the controller matrix $K$ thus contains the coupling from the error $e$ to the control signal $u_{m}$. Figure 1 shows the closed-loop system with the signal path $u_{m}$ broken. Any sensible choice of the controller row $k$ that improves the performance of loop $m$, requires at least knowledge of the SIMO transfer matrix from $u_{m}$ to $e$ in this partially open system. We denote this transfer matrix $H=-\left(I+G_{1} K_{1}\right)^{-1} G_{2}$ and assume that it is stable. The block diagram of Figure 2 shows explicitly the contribution of controller row $m$ to the feedback control of the system. The transfer matrices of the full multivariable closed-loop system can easily be described in terms of those for the system with $H$ acting as a process and $k$ as a controller. In other words, the multivariable control design problem for $G$ is reduced to a SIMO control problem for $H$ with MISO controller $k$.

\section{Parameterization and stability}

It is simple to calculate the effect of new or redesigned controller row elements of the single-loop opening ap-

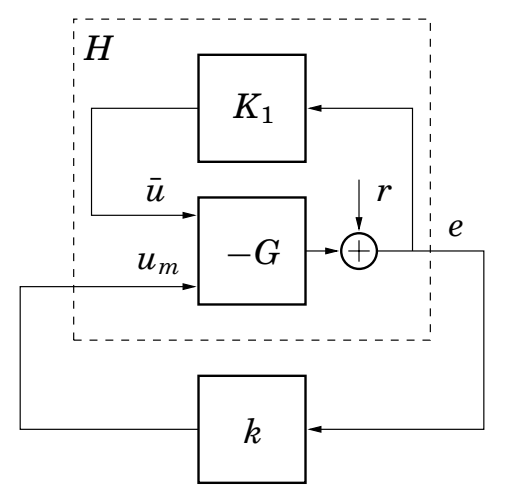

Figure 2 Contribution of controller row $k$.

proach. The input sensitivity function is given by $S_{i}:=(I+K G)^{-1}$ and the output sensitivity function by $S_{o}:=(I+G K)^{-1}$. The diagonal element $m$ of the sensitivity matrix $S_{i}$ captures much of the performance in loop $m$. By the definition of $H$ and $k$, we have $\varepsilon_{m}^{T} S_{i} \varepsilon_{m}=1 /(1-k H)$. Knowledge of $H$ alone is thus sufficient to compute the transfer function for loop $m$ that results from a particular choice of $k$.

The closed-loop transfer matrices are affine functions in the Youla parameter $Q:=(I+K G)^{-1} K$ if $G$ is stable [11]. For example, the sensitivity and complementary sensitivity matrices with reference to process inputs are $S_{i}=I-Q G$ and $T_{i}=Q G$, respectively, and the corresponding matrices with reference to process outputs are $S_{o}=I-G Q$ and $T_{o}=G Q$. The closedloop transfer matrices are also affine functions in $q:=$ $k /(1-k H)$. This $1 \times m$ vector of transfer functions is the Youla parameter for the partially open system. Some calculations give the relation between $q$ and $Q$ as

$$
Q=\left(\begin{array}{c}
K_{1} \\
0
\end{array}\right)\left(I+G_{1} K_{1}\right)^{-1}+\left(\begin{array}{c}
K_{1} H \\
1
\end{array}\right) q\left(I+G_{1} K_{1}\right)^{-1} .
$$

Parameterization of stabilizing controller rows and columns are studied in [8].

Naturally, any adjustments of controller row $m$ must be made in such a way that the closed-loop system remains stable. The following result follows from the Nyquist theorem. Assume the closed-loop system is stable with controller row $k$. Let $k$ be replaced by $\widehat{k}$, where $\widehat{k}$ is such that no unstable modes are cancelled and that the number of open-loop RHP poles does not change. Then the adjusted closed-loop system remains stable if and only if $\mathcal{N}(1-\widehat{k} H, 0)=\mathcal{N}(1-$ $k H, 0)$, where $\mathcal{N}(f(s), z)$ is the number of clockwise encirclements of the point $z$ by the image of the usual Nyquist contour $D_{\mathcal{X}}$ under the map $f$ as it is traversed in a clockwise direction. 


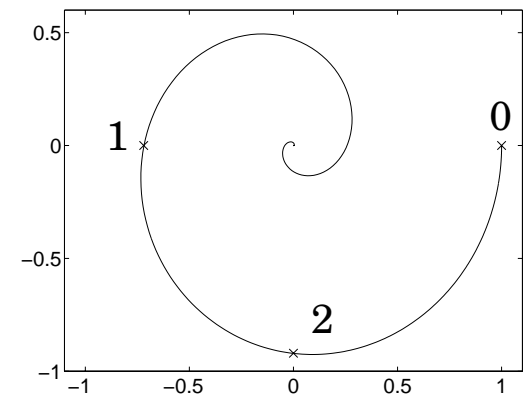

Figure 3 Three important points on the Nyquist curve.

\section{Extended Relay Experiment}

A relay feedback experiment is a simple and robust way of doing closed-loop identification. The setup for the original SISO experiment is simply to replace the SISO controller by a relay [1]. The main advantages of an identification experiment based on relay feedback are (1) that the frequency of the excitation signal is near the cross-over frequency of the open-loop system, (2) that the experiment is done in closed loop, and (3) that no prior knowledge about the process dynamics is needed. The frequency of the relay output is close to optimum in the sense that it is in the band where the estimated model has to be accurate to support a satisfying control design. Even if no controller is present in the loop during the experiment, the relay itself gives a high-gain feedback. This means, for instance, that the process is automatically kept close to its operating point during the experiment.

A drawback with the original relay feedback experiment is in some cases its lack of excitation. Therefore, we introduce a modification of the standard relay experiment, by simply estimating two points on the Nyquist curve instead of one. It is well-known that with a filter in series with the relay, any point on the Nyquist curve can be estimated [2]. This idea has been explored for SISO systems in $[15,16]$. Persson [13] investigated the amount of process information needed for control design in number of points and their location on the Nyquist curve. Three crucial points are marked with crosses in Figure 3. Point 1 is determined by a standard relay experiment, whereas Point 2 is determined from an experiment with a relay and an integrator in series. The method can be interpreted as putting a filter $W$ in series with the relay. The filter is initially set to $W=1$ and then to $W=1 / \mathrm{s}$. Together with steady-state data, the gained information is sufficient to derive a model of the form

$$
G(s)=\frac{b_{0} s+b_{1}}{s^{3}+a_{1} s^{2}+a_{2} s+a_{3}} .
$$

The controller tuning described in Section 2 is based on knowledge of the column vector $H$. The set-up for

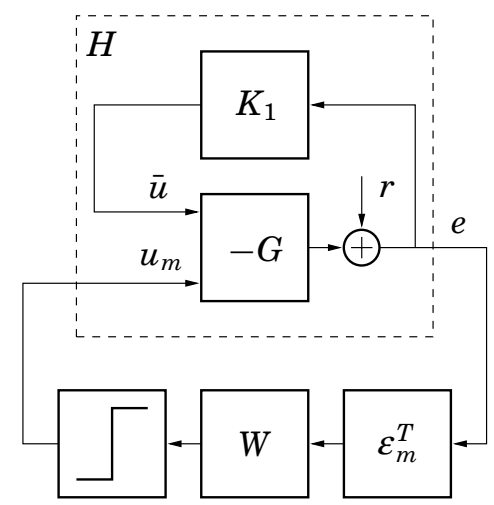

Figure 4 Relay experiment for identifying $H$.

an extended relay experiment to identify $H$ is shown in Figure 4, compare with Figure 2. The block with $\varepsilon_{m}^{T}$ picks out error signal $e_{m}$. The relay is thus connected between $W e_{m}$ and $u_{m}$. This gives an oscillation with frequencies determined by $H_{m}$, which is typically the most important transfer function for controller tuning in loop $m$. From measuring $\bar{e}$ and $e_{m}$, we can estimate all elements of $H$. We summarize the method in the following algorithm.

\section{ALGORITHM 1-SIMO RELAY EXPERIMENT}

1. Set $W=1$ and wait for a stationary oscillation. Measure the frequency $\omega_{1}$ and derive the response for each element $H_{i}$.

2 . Set $W=1 / s$ and wait for a stationary oscillation. Measure the frequency $\omega_{2}$ and derive the response for each element $H_{i}$.

3. Freeze the relay output and wait for steadystate and derive the steady-state gains for each element $H_{i}$.

4. Estimate $H_{i}$ as in (2) based on the responses and the corresponding frequencies $\omega_{1}$ and $\omega_{2}$.

The amounts of time required for a stationary oscillation in Step 1 and Step 2 are small. Experiments show that stationarity is often reached after three-four relay switches.

Note that Algorithm 1 automatically gives highest priority to the last element of $H$ in the sense that the excitation frequencies are adjusted to suit $H_{m}$. This means also that if $H_{1}, \ldots, H_{m-1}$ give small responses around the cross-over frequency of $H_{m}$, then the estimates of $H_{1}, \ldots, H_{m-1}$ are probably poor. However, because the elements are small, the lack of accuracy has only a small influence on the control performance. 

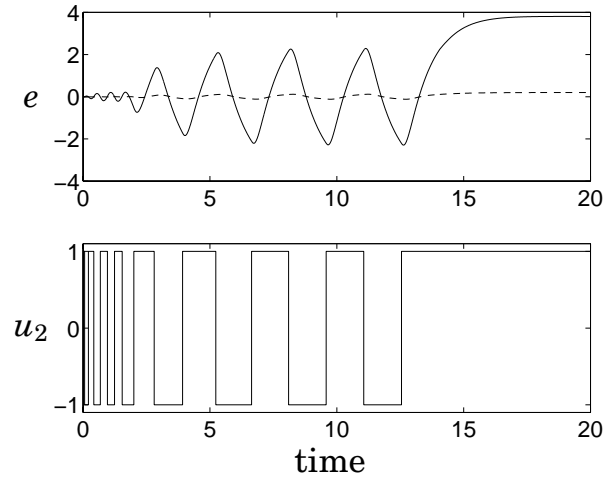

Figure 5 Extended relay experiment for minimum phase system. The error signal $e_{1}$ (dashed) is negligible compared to $e_{2}$ (solid).

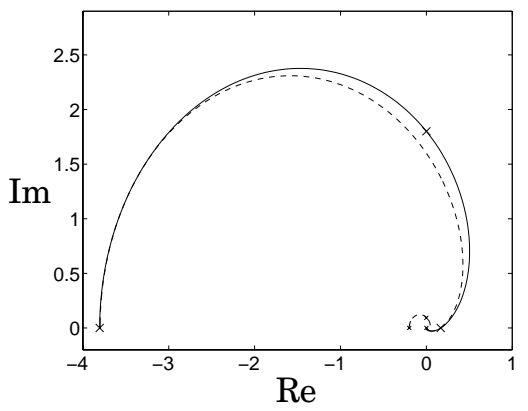

Figure 6 Nyquist curves of $H$ for minimum phase system. The crosses are estimated frequency points from relay feedback experiments. The small crosses correspond to $H_{1}$ and the large to $\mathrm{H}_{2}$. A third-order estimate of $\mathrm{H}_{2}$ is also shown (solid line). The frequency response of $H_{1}$ is negligible compared to the response of $\mathrm{H}_{2}$.

\section{Example}

In this section the retuning procedure is applied to a multivariable level control problem. The considered system is a normalized model of the quadruple-tank laboratory process described in $[9,10]{ }^{1}$ The system including models for actuators and sensors is given by

$$
G=\frac{500}{(s+10)^{2}}\left(\begin{array}{cc}
\frac{\gamma_{1}}{s+1} & \frac{1-\gamma_{2}}{(s+1)^{2}} \\
\frac{1-\gamma_{1}}{(s+1)^{2}} & \frac{\gamma_{2}}{s+1}
\end{array}\right) .
$$

The parameters $\gamma_{1}, \gamma_{2} \in[0,1]$ are determined by how two valves are set prior to an experiment. The system $G$ has a RHP zero if and only if $\gamma_{1}+\gamma_{2} \in(0,1]$. Next we study the system for one minimum-phase setting and one nonminimum-phase setting.

Minimum phase system Let $\gamma_{1}=\gamma_{2}=4 / 5$. Then $G$ has zeros in $-5 / 4$ and $-3 / 4$, so the system is minimum

\footnotetext{
${ }^{1}$ The retuning procedure in this paper has been applied to the real laboratory process in [14].
}
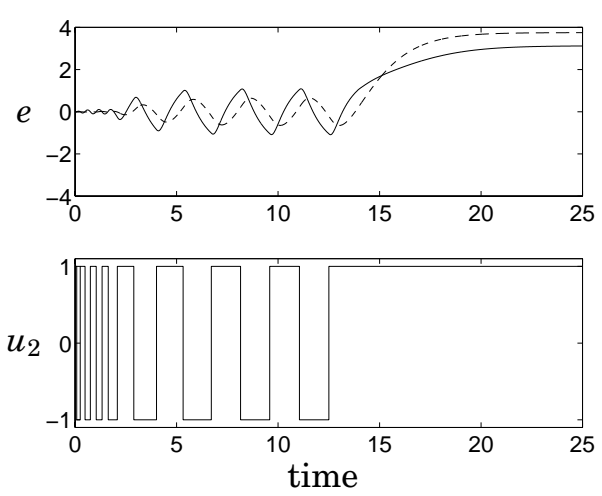

Figure 7 Extended relay experiment for nonminimum phase system. The error signals $e_{1}$ (dashed) and $e_{2}$ (solid) are of the same magnitude.

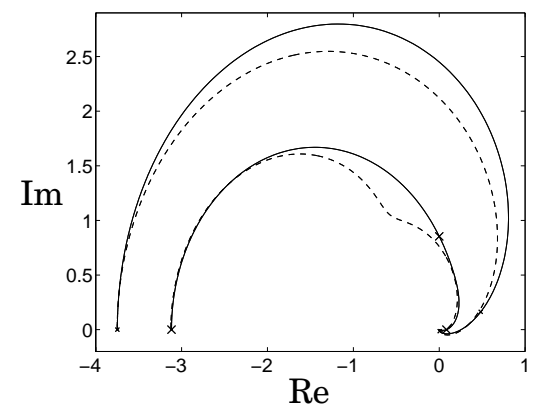

Figure 8 Nyquist curves of $H$ for nonminimum phase system, compare Figure 6 . The frequency responses of $H_{1}$ and $H_{2}$ are of the same magnitude.

phase. Let $K=\operatorname{diag}\{1,1\}$ be the initial controller. The response of the extended relay experiment described in Algorithm 1 is shown in Figure 5. The response of $e_{1}$ is small compared to $e_{2}$. This is further illustrated in Figure 6, where the small crosses are the estimated frequency points for $H_{1}$ and the large crosses the points for $\mathrm{H}_{2}$. The dashed curves are the Nyquist curves for the true systems, whereas the solid curve is a third-order estimate of $\mathrm{H}_{2}$.

The result from the relay experiment indicates that we can neglect the influence of $H_{1}$ and simply retune the last element of $k$. The PI controller $k=(0,(2 s+$ $3) / s$ ) gives the poles -41.9 and $-2.2 \pm 4.6 i$ for the second diagonal element of $S_{i}$. Note that the tuning here corresponds to applying SISO methods. For this example the MIMO characteristics of the system are insignificant.

Nonminimum phase system Let us now change the valves so that $\gamma_{1}=\gamma_{2}=2 / 5$. Then $G$ has zeros in $-5 / 2$ and $1 / 2$, so the system is nonminimum phase. Let $K=\operatorname{diag}\{-0.1,0.1\}$ be the initial controller. Figure 7 shows the result of the relay experiment. The estimated Nyquist curves (solid) are shown in Figure 8 , together with the true ones (dashed). We 
see that the interaction is severe, so it is probably not sufficient to only retune the second loop. If a relay experiment is also done in the first loop, it is straightforward to derive a multivariable controller, for example based on decoupling.

\section{Conclusions}

It was shown how a poorly tuned multivariable controller can be retuned through a simple closed-loop experiment based on relay feedback and controller row design. In particular, the case with one bad loop was discussed. The standard SISO relay feedback experiment in [2] was extended to give better excitation and a more accurate model, which seems to be necessary for many MIMO control designs. Several results on how a row in the controller matrix affects the closed-loop performance were derived. No fully automatic procedure was described in the sense of automatic tuning for SISO systems. It is believed that this can only be done if the considered class of systems is more limited than in this paper. It was pointed out through an example that for "simple" multivariable control systems the proposed method agrees with automatic SISO tuning. For "difficult" MIMO control problems the method still provides a solid ground for controller design.

\section{References}

[1] K. J. Åström and T. HÄGGLUnd. "Automatic tuning of simple regulators with specifications on phase and amplitude margins.” Automatica, 20, pp. 645-651, 1984.

[2] K. J. Åström and T. HÄGGLund. PID Controllers: Theory, Design, and Tuning. Instrument Society of America, Research Triangle Park, NC, second edition, 1995.

[3] K. J. Åström, T. H. LeE, K. K. TAN, and K. H. JohansSON. "Recent advances in relay feedback methods-a survey.” In IEEE SMC Conference, pp. 2616-2621, Vancouver, WA, 1995. Invited paper.

[4] W. L. BiALKowski. "Dreams vs reality: A view from both sides of the gap." In Control Systems '92, Whistler, B.C., Canada, 1992.

[5] D. B. EndER. "Process control performance: Not as good as you think." Control Engineering, 40:10, pp. 180-190, 1993.

[6] M. FRIMAN and K. V. WALLER. "Autotuning of multiloop control systems.” Ind. Eng. Chem. Res., 33, pp. 17081717, 1994.

[7] C. C. Hang, A. P. LoH, and V. U. Vasnani. "Relay feedback auto-tuning of cascade controllers." IEEE Trans. on Control Systems Technology, 2:1, pp. 42-45, 1994.

[8] B. JAMES and G. F. BRYANT. "A parametrization for automatic loop-by-loop multivariable controller design."
In 34th IEEE Conference on Decision and Control, New Orleans, LA, 1995.

[9] K. H. Johansson. Relay feedback and multivariable control. PhD thesis ISRN LUTFD2/TFRT--1048--SE, Department of Automatic Control, Lund Institute of Technology, Lund, Sweden, November 1997.

[10] K. H. JOHANSSON and J. L. R. NuNEs. "A multivariable laboratory process with an adjustable zero." In 17th American Control Conference, Philadelphia, PA, 1998.

[11] J. M. MACIEJowski. Multivariable Feedback Design. Addison-Wesley, Reading, MA, 1989.

[12] Z. J. Palmor, Y. Halevi, and N. Krasney. "Automatic tuning of decentralized PID controllers for TITO processes." Automatica, 31:7, pp. 1001-1010, 1995.

[13] P. Persson. Towards Autonomous PID Control. PhD thesis ISRN LUTFD2/TFRT--1037--SE, Department of Automatic Control, Lund Institute of Technology, Lund, Sweden, April 1992.

[14] V. RECICA. "Automatic tuning of multivariable controllers." Master thesis ISRN LUTFD2/TFRT--5592-SE, Department of Automatic Control, Lund Institute of Technology, Lund, Sweden, 1998.

[15] T. S. SchEI. "A method for closed loop automatic tuning of PID controllers." Automatica, 28:3, pp. 587-591, 1992.

[16] T. S. ScHEI. "Automatic tuning of PID controllers based on transfer function estimation." Automatica, 30:12, pp. 1983-1989, 1994.

[17] S.-H. SHEN and C.-C. YU. "Use of relay-feedback test for automatic tuning of multivariable systems." AIChE Journal, 40:4, pp. 627-646, 1994.

[18] F. G. SHINskey. Controlling Multivariable Processes. Instrument Society of America, Research Triangle Park, NC, 1981.

[19] V. U. Vasnani. Towards Relay Feedback Auto-Tuning of Multi-Loop Systems. PhD thesis, National University of Singapore, 1994.

[20] Q. G. Wang, B. Zou, T. H. LeE, and Q. BI. "Auto-tuning of multivariable PID controllers from decentralized relay feedback." Automatica, 33:3, pp. 319-330, 1997.

[21] P. Zgorzelski, H. Unbehauen, and A. NiederLinski. "A new simple decentralized adaptive multivariable regulator and its application to multivariable plants." In IFAC 11th Triennial World Congress, pp. 381-386, Tallinn, Estonia, 1990.

[22] M. ZhuAnG and D. P. Atherton. "Optimum cascade PID controller design for SISO systems." In IEE Control '94, pp. 606-611, Warwick, 1994.

[23] M. Zhuang and D. P. Atherton. "PID controller design for a TITO system." IEE Proc. Control Theory Appl., 141:2, pp. 111-120, 1994. 